HIGHER SELF-EFFICACY ASSOCIATED WITH LOWER INTERLEUKIN-6 IN OLDER ADULTS WITH CHRONIC DISEASE

M. Hladek ${ }^{1}$, J. Gill ${ }^{2}$, C. Lai ${ }^{2}$, S. Szanton ${ }^{3}$, 1. Johns Hopkins University, 2. National Institute of Health, National Institute of Nursing Research, 3. Johns Hopkins School of Nursing

Although inflammation is common in older adults with chronic diseases, not all have the same amount or degree of poor health outcomes from the inflammation or chronic diseases. Self-efficacy is integral to successful disease management but it is unclear if and how this relates to inflammation. The purpose of this study was to evaluate associations between self-efficacy and Interleukin-6 (IL-6), which is an inflammatory cytokine highly associated with all-cause morbidity and mortality. We conducted a two-visit cross-sectional study with 161 community dwelling adults ages 65 and older in Maryland. All participants had at least one chronic condition (e.g. hypertension, diabetes, arthritis, etc.) Those with a terminal illness diagnosis, active cancer treatment, cognitive impairment or progressive neurological condition were excluded. Sweat IL-6, self-efficacy, number of chronic diseases, social support and other socio-demographic data were collected. Sweat was collected using a non-invasive sweat patch worn for 72 hours by each participant. We examined the effect of self-efficacy in predicting sweat IL-6 after controlling for co-variates using multiple linear regression. There was a significant relationship between self-efficacy and IL-6 $(\beta=-.02 . p=0.004)$ that remained significant after adjustment for age, sex, race, body mass index, number of chronic conditions and social support $(\beta=-0.22, \mathrm{p}=0.005)$. Higher self-efficacy was significantly related to lower IL-6. Although preliminary, this could shed light on the mechanistic connection between psychological and physical states. Enhancing self-efficacy might also be a viable non-pharmacological treatment to lower or slow inflammatory burden in older adults.

\section{HIGHER SERUM LEVELS OF FIBROBLAST GROWTH FACTOR 21 IN GERIATRIC PATIENTS WITH CACHEXIA}

K. Franz ${ }^{1}$, M. Ost ${ }^{2}$, C. Herpich ${ }^{3}$, L. Otten ${ }^{1}$, V. Coleman ${ }^{2}$, S. Klaus ${ }^{2}$, U. Müller-Werdan ${ }^{4}$, K. Norman ${ }^{5}$, 1. Charité - Universitätsmedizin Berlin, Nutrition and Body Composition - Research Group on Geriatrics (FGG), Berlin, Germany, 2. German Institute of Human Nutrition Potsdam-Rehbrücke, Dept. of Physiology and Energy Metabolism, 3. German Institute of Human Nutrition Potsdam-Rehbrücke, Dept. of Nutrition and Gerontology, 4. Geriatrics Research Group, Charité - Universitätmedizin Berlin, 5. Institute of Human Nutrition Potsdam-

Rebbrücke, Dept. of Nutrition and Gerontology, Germany

The hormonal mediator fibroblast growth factor 21 (FGF21) is promptly induced by short fasting periods in animal models to regulate glucose and fat metabolism (Inagaki et al. 2007). Data on FGF21 in humans are controversial and FGF21 has not yet been investigated in geriatric patients with loss of appetite and ongoing weight loss. We aimed to explore the association of FGF21 with cachexia in old patients and a healthy control group. Serum FGF21 levels were measured with commercial ELISA-kits. Cachexia was defined as $\geq 5 \%$ weight loss in the last three months (Evans et al., 2008) and concurrent anorexia (Council on Nutrition appetite questionnaire). We included 103 patients (76.9 \pm 5.2 years, $53.4 \%$ women) and 56 healthy controls (72.9 \pm 5.9 years, $57.1 \%$ women) in a prospective cross-sectional study. Cachectic patients $(\mathrm{n}=17,16.5 \%)$ had significantly higher FGF21 levels compared to non-cachectic patients $(952.1 \pm 227.8 \mathrm{pg} / \mathrm{ml} \mathrm{vs}$. $525.2 \pm 66 \mathrm{pg} / \mathrm{ml} ; \mathrm{p}=0.022)$. The lowest FGF21 levels were found in old healthy controls $(293.3 \pm 20.5 \mathrm{pg} / \mathrm{ml}$, global $\mathrm{p}<0.001)$. Overall in the study cohort, percentage of weight loss $(\mathrm{r}=0.442, \mathrm{p}=0.001)$ and loss of appetite $(\mathrm{r}=-0.277$, $\mathrm{p}=0.001)$ correlated significantly with FGF21 levels, whereas there was no correlation between overall BMI and FGF21 (r= - 0.148, $\mathrm{p}=0.081$ ). Geriatric patients with cachexia and anorexia exhibited the highest FGF21 levels. Furthermore, there was a significant correlation between the degree of weight loss, anorexia and FGF21 levels. Whether this is an adaptive response to the nutrient deprivation in disease-related cachexia or the increased FGF21 values contribute to the catabolic state, needs to be clarified.

\section{MULTIMODAL STUDY OF GERIATRIC MILD TRAUMATIC BRAIN INJURY USING COMPUTED TOMOGRAPHY AND MAGNETIC RESONANCE IMAGING}

A. Irimia, A. Maher, N. Chowdhury, K. Rostowsky, M. Law, University of Southern California

In recent decades, the joint use of computed tomography (CT) and magnetic resonance imaging (MRI) to study geriatric mild traumatic brain injury (mTBI) has increased considerably. Although MRI is the gold standard for the noninvasive evaluation of white matter (WM) and gray matter (GM) volumes in the living human brain, CT continues to be used widely during treatment of geriatric mTBI, particularly when MRI is unavailable or contraindicated. Furthermore, in clinical settings, CT scans are frequently acquired from geriatric mTBI patients even though MRI scans are not. Partly for this reason, there has been renewed interest in the development of CT-only approaches to the calculation of brain structure changes after geriatric mTBI. In this study, we introduce a novel, CT-only brain segmentation method and validate it using both MRI and CT volumes acquired from ten geriatric mTBI volunteers (age: $\mu=65$ yrs; $\sigma=7$ yrs). Segmentation accuracy is quantified using the SørensenDice coefficient (SDC), a robust measure of inter-modality tissue classification agreement between CT and MRI. Across all cases, the mean and standard deviation of the SCD is found to be $85.5 \% \pm 4.6 \%$ for WM and $86.7 \% \pm 5.6 \%$ for GM, indicating excellent ability to calculate GM/WM volumes from CT in geriatric mTBI patients. Since mTBI has higher incidence after age 65 than in any other adult age group, our contribution is important because it broadens the ability to integrate CT-based findings into research studies of geriatric mTBI and of its effects upon brain aging and neurodegeneration.

\section{SEIZURES IN ALZHEIMER'S DISEASE ARE ASSOCIATED WITH YOUNGER ONSET AND DECREASED LIFE EXPECTANCY}

L. Baumann 1 , S. Sullivan 2 , S. Shirk ${ }^{3}$, L. Moo ${ }^{4}$, 1. Bedford

VAMC, William James College, 2. Bedford VAMC, 3. 